\title{
LED: A Fast Overlapping Communities Detection Algorithm Based on Structural Clustering
}

\author{
Tinghuai Ma ${ }^{\mathrm{a}, *}$, Yao Wang ${ }^{\mathrm{b}}$, Meili Tang ${ }^{\mathrm{c}}$, Jie Cao ${ }^{\mathrm{d}}$, Yuan Tian ${ }^{\mathrm{e}}$, Abdullah Al-Dhelaan ${ }^{\mathrm{e}}$, \\ Mznah Al-Rodhaan ${ }^{\mathrm{e}}$ \\ ${ }^{a}$ Jiangsu Engineering Center of Network Monitoring, Nanjing University of information science $\mathcal{E}$ Technology, Nanjing 210-044,China \\ ${ }^{b}$ School of Computer $\mathcal{E}$ Software, Nanjing University of information science $\mathcal{E}$ Technology, Jiangsu, Nanjing 210-044, China \\ ${ }^{c}$ School of Public Administration, Nanjing University of Information Science $\mathcal{E}$ Technology, Nanjing 210-044, China \\ ${ }^{d}$ School of Economics $\mathcal{E}$ Management,Nanjing University of Information Science $\mathcal{E}$ Technology, Nanjing 210-044, China \\ ${ }^{e}$ Computer Science Department, College of Computer and Information Science, King Saud University, Riyadh 11362, Saudi Arabia
}

\begin{abstract}
Community detection in social networks is a fundamental task of complex network analysis. Community is usually regarded as a functional unit. Networks in real world more or less have overlapping community structure while traditional community detection algorithms assume that one vertex can only belong to one community. This paper proposes an efficient overlapping community detection algorithm named LED (Loop Edges Delete). LED algorithm is based on Structural Clustering, which converts structural similarity between vertices to weights of network. The evaluations of the LED algorithm are conducted both from classical networks from literature and C-DBLP, which is a huge and real-life co-author social network in China. The results show LED is superior to other methods in accuracy, efficiency, comparing with FastModurity and GN algorithm.
\end{abstract}

Keywords: Overlapping Communities, Structural Similarity, C-DBLP

\section{Introduction}

Many dataset can be represented as networks. Networks are usually modeled as a graph $G(V, E)$, where $V$ is a set of vertices and $E$ is a set of edges. For example, a online social network can be modeled as a graph, where users are represented as vertices that are connected by an edge when one user follows another. Community structure[1] is a common property of many networks. In terms of our experience on online social networks, it's a common sense that people with same interests trending to get together as communities: subsets of vertices within which vertex to vertex connections are dense, but between which connections are sparse[2, 3, 5, 6]. Also, some active members of social network may take part in many communities simultaneously, which in networks we call them overlapping vertices.

Network clustering (or graph partitioning) is aim at detecting community structure. Community detection is an useful tool to mine the hidden information from networks. Xu Y et al.[7] proposed a community forest model which takes a community as a tree. A tree consists of trunks and leaves, while a community consists of vertices and relations. Strong relations are like the tree trunk, some core vertices are like the tree roots and some border vertices are like the leaves. Community detection is just like finding trees from the forest. Overlapping is a common phenomenon in networks, overlapping community detection takes this factor into count detecting overlapping or hierarchical communities.

\footnotetext{
${ }^{*}$ Corresponding author: thma@ @uist.edu.cn
} 
Nowadays we are in the era of information explosion, networks are getting larger and larger. We need much more efficient communities detection algorithms to analyze network with millions of vertices. In this paper, we propose a new method for very large networks which has linear time complexity. The time complexity of our algorithm is linear, only a few state of the art algorithms[9, 10] can do this. The goal of our algorithm is to find communities and overlapping vertices in very large networks. To achieve this goal, our algorithm must be fast and precise. Our algorithm is inspired by density-based algorithm DBSCAN[4]. We transfer the structure relationship between vertices to their weight by computing their structural similarity. Structural similarity represents connection strength of two vertices, because it counts all common neighbors the two vertices share. It is a common sense that two people who share many friends should be clustered in the same community. We delete the edges of small weights to disconnect vertices which should not be clustered in the same community. From the deleted edges, the initial communities are formed. Our algorithm only needs to traverse all the edges of the network for once, calculating the structure similarity of the edge's two vertices and deleting the edges with structure similarity below the threshold $\alpha$ (Manually specified).

In many real world networks, the overlapping communities are universal[25]. For example, the network in Figure 1 , it is a simple network, so we can artificial determine that it consists of two communities and one overlapping verte $x$. It is divided into two communities by GN algorithm[11]. Vertices from vertex 1 to vertex 6 are at one community, the rest of vertices are within another community. Infomap algorithm[12] gives exactly the same partition. Apparently, the vertex 7 connects to the two communities with almost the same strength. Vertex7 should be regard as member of two communities at the same time. Algorithms we mentioned before don't have the ability to identify overlapping vertices while we extend the ability to LED. The overlapping vertex often play an important role in network. As we all know, community is regarded as functional unit in network. The overlapping vertices promote the interaction between functional units. In real networks, overlapping vertex is useful in web search engine optimization[13, 16], viral marketing[14], epidemiology[15] and so on.

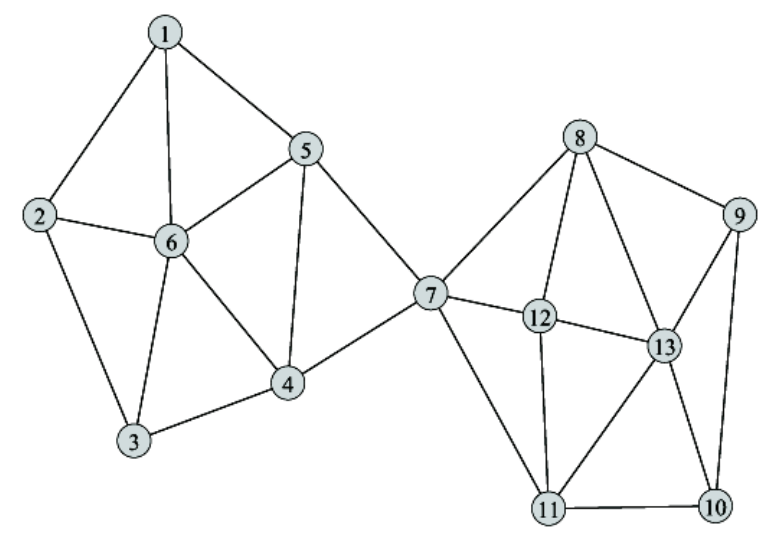

Figure 1: A Network with two Communities and one Overlapping Vertex

The paper is organized as follows. In Section 2, we introduce the related work of overlapping community detection. Section 3 describes the algorithm we propose, including Structural Similarity, Loop Edges Delete Process, Overlapping Vertices Detection and LED Algorithm. In Section 4 we analysis the time complexity of our algorithm in theory and the selection of parameter. In Section 5, we run our algorithm on C-DBLP and other real world social networks. Finally, we present our conclusions and future work in Section 6.

\section{Related Work}

In this section we survey related works firstly and then give a toy motivating example. 


\subsection{Related Work}

Community detection is a fundamental task of complex networks analysis. It has great contribution to other studies such as recommender system[17] and privacy protection[18]. A great deal of work has been done to detect communities in complex networks, they can be categorized into two classes according to whether allow overlapping: disjoint community detection and overlapping community detection.

In early studies of community detection, researchers rarely took the overlapping community into their definition of community. Proposing a measure of quality of community and then using a greed algorithm to maximize or maximize the measure is an important kind of disjoint community detection algorithms. The GN algorithm[11] which is probably the best-known algorithm for finding community structure is based on the betweenness centrality measure[26]. Structure entropy[24] is an information theoretical measure of complexity of networks. Justine et al.[25] proposed local neighborhood ratio to define community structure. They all have similar greed algorithms but with different measures. CONGA[23], proposed by Steve Gregory, is based on Girvan and Newman's algorithm[11]. On GN algorithm's basis, CONGA extends a novel method of splitting vertices for allowing overlapping communities. It performs well on randomly generated networks and has interesting results on a range of real-world networks. It is based on an old algorithm, so it is not efficient enough for large networks. One year later, Steve Gregory proposed a new algorithm named CONGO[27] (CONGA Optimized). GN algorithm and CONGA both relay on betweenness which is a global centrality measure. In CONGO, Steve Gregory refined the global betweenness to a local betweenness which greatly reduces the time complexity.

In recent years, overlapping community detection have attracted more and more attention. The area of overlapping community detection is the hot area currently. Clique percolation method $(C P M)$ [19] may be the most famous and widely used overlapping community detection algorithm which is based on graph theory[20]. CPM claims "The basic observation on which our community definition relies is that a typical community consists of several complete (fully connected) subgraphs that tend to share many of their nodes." CPM finds $k$-cliques( $k$ fully connected subgraphs) and the $k$-cliques's series of adjacent $k$-cliques (where adjacency means two $k$-cliques sharing $k$ - 1 nodes) to detect overlapping community. However, CPM has many problems. It has an inappropriate community definition to detect overlapping community for different kinds of networks. Although CPM can adapt to different networks by selecting a different $k$, it has only limited effects. When the network is too dense, CPM detects giant communities. When the network is too sparse, it finds no communities at all. Also it is a very time-consuming algorithm, in theory, its time complexity is $O\left(n^{3}\right)$.

Yong-Yeol A el al.[21] considered community to be a set of closely connected links instead of vertices with many links between them. The link communities incorporate overlapping vertices as vertices can belong to many links of different communities. In general, the number of links is much more than the number of vertices, so it is a more timeconsuming algorithm. Brian B el al.[22] proposed a probabilistic model of link communities to detect communities, either overlapping or not, and used a fast, closed-form expectationCmaximization algorithm to analyze networks of millions of vertices in reasonable running time.

There are many other tries to detect overlapping communities. Du et al.[28] use the maximal cliques for community detection. The algorithm finds all the maximal cliques as the initial communities, then assigns the rest vertices to the closest initial community. EAGLE[29] and GCE[30] also work this way. Our algorithm's main process is similar to theirs, but we don't use cliques to get initial communities. BELP, proposed by Chen et al.[33], is based on SLPA [34] (Speaker-listener Label Propagation Algorithm) which is a unstable algorithm. For edges connecting two communities, it uses average degree to make decision for which community the vertices should belong to. This strategy inspires us. We use a similar strategy to identify overlapping vertices. Embedding is another popular way to perform community detection. SSDE-Cluster[31] first embeds the graph and then performs a metric clustering using a Gaussian Mixture Model $(G M M)$. Creating an approximate embedding is useful for clustering in metric spaces and GMM can be adapted to get overlapping communities. Fuzzy clustering algorithm[32] proposes the combination of a new modularity function based on generalizing Newman's $Q$ function. It uses spectral method mapping vertices of the networks into Euclidean space. Then a fuzzy $c$-means divides the network into communities.

\subsection{Motivating Example}

Here is a toy example for our algorithm. It explains why our methods is more reasonable. For network in Fig.1, our algorithm computes the structural similarity of each edge and the weighted network is shown in Fig.2. The structural 


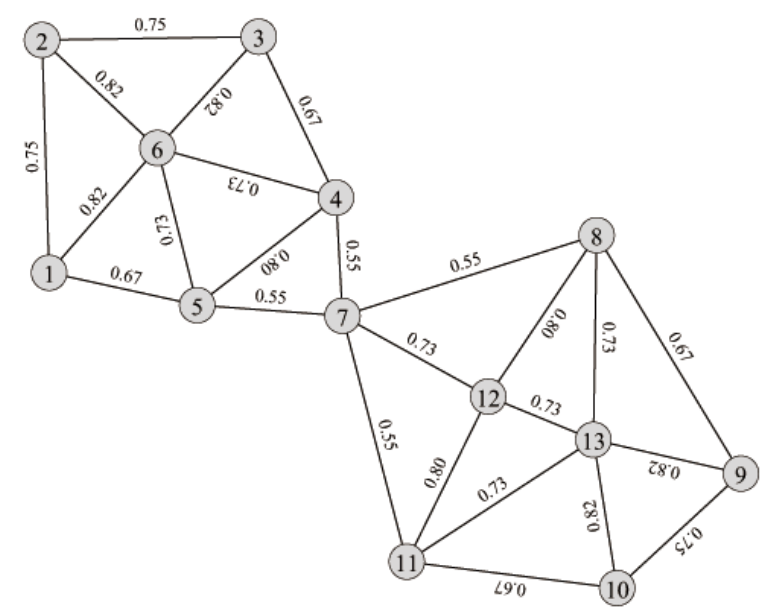

Figure 2: A Network with structural similarities as weights

similarities of $e(4,7), e(5,7), e(7,8)$ and $e(7,11)$ are smallest of all. They certainly will be deleted and the deletion of $e(4,7)$ and $e(5,7)$ will cause the network splits into two communities. With proper threshold our algorithm divides it into two communities with the same result as algorithms mentioned before. Our algorithm detects overlapping vertices by testing vertex $4,5,7$ with a good judgment[35] (details will be discussed in Section3.3). Vertex7 is detected as a overlapping vertex by our algorithm in this network. So, our algorithm detects two communities, community $1\{1,2$, $3,4,5,6,7\}$, community $2\{7,8,9,10,11,12,13\}$, and vertex 7 is the overlapping vertex of the two communities.

\section{LED: Loop Edges Delete Algorithm}

The LED algorithm is classified into three steps. Firstly, the structural similarity of two vertices is calculated as weights of their edge. Secondly, the weightless edges are deleted. Then, the overlapping vertices are detected. At last the isolated vertices are reconnected and find the overlapping communities.

\subsection{Structural Similarity}

In this paper, we focus on undirected and unweighted graph. Let $G(V, E)$ be a graph, where $V$ is a set of vertices and $E$ is a set of pairs of distinct vertices, called edges. The local structure around a vertex can be expressed by its neighborhood. A formal definition of vertex structure is given as follows.

Definition 1 (Vertex Structure)

Let $v \in V$, the structure of $v$ is defined by its neighborhood, donated by $\Gamma(v)$,

$$
\Gamma(v)=\{w \in V \mid(v, w) \in E\} \cup\{v\}
$$

Then, we define the structural similarity based on this intuition that the more friends of two people in common, the more probability they are friends. The simplest method to define the structural similarity is using the number of neighbors of two vertices in common. The range of structural similarity will be uncertain and change with different networks. Therefore, we normalize the number of common neighbors by the geometric mean of the two neighborhoods' size.

Definition 2 (Structural Similarity)

$$
\sigma(u, v)=\frac{|\Gamma(u) \cap \Gamma(v)|}{\sqrt{|\Gamma(u)| *|\Gamma(v)|}}
$$


The formula above is based on the common sense that two people who share more friends should be closer. Any other similarity measures which can embody this common sense such as Jaccard[37] or Dice[36] are suitable. The structural similarity of two vertices indicates the strength of their relationship, while weights of edges have the same meaning. In our algorithm, the structural similarity of two vertices is computed to be their weight. The structural similarity transfers the structure relationships of two vertices to their weight for unweighted graph. This method makes the structure of networks quantized. Further, for a given vertex $v$ we define its $\alpha-$ neighborhood as follows.

Definition 3 ( $\alpha-$ neighborhood)

$$
N_{\alpha}(v)=\{w \in \Gamma(v) \mid \sigma(v, w) \geq \alpha\}
$$

It extracts important relationships from a single vertex. For the whole graph, the $\alpha-$ neighborhoods form core structure of the graph.

\subsection{Loop Edges Delete Process}

The Loop Edges Delete Process means extracting the core structure from graph. The connected relation of core vertices can be seen as initial communities. For the given threshold $\alpha$, we remove the edges weighted lower than $\alpha$, then we get some separated parts. But delete edges once may not be enough. For some small $\alpha$, the networks may extract out large inter-connected components. To eliminate sensitive to threshold and get better initial communities, we adopt an operation called LED (Loop Edges Delete). For every inter-connected components, compute the structural similarity and with the same $\alpha$ execute the edge deleting process mentioned above, then we get more and smaller inter-connected components. The Loop Edges Delete process will be loop executed until no edge is deleted. When the threshold is set small, the first edges delete process will get giant initial communities, but the following edges delete process will divide the giant communities into proper size.

We use the well-known Zachary's "karate club" dataset[38] to explain how to use structural similarity for community detection. In that study, Zachary observed a karate club with 34 members for two years. During that time, the administrator of the club and the club's instructor had a conflict between. The instructor end up left the karate club and started a new club. Figure 3(a) shows the Karate network, with the instructor and the administrator represented by vertex 1 and vertex 34 , respectively. The weights of edges are generated by calculating their structural similarities using formula(2). We set the threshold $\alpha=0.5$. We delete the edges whose weights are lower than 0.5 and then we get the network in Figure 3(b). In other words, Our algorithm keep edges between vertex and its 0.5 - neighborhood then we get four inter-connected components. By looking into the four inter-connected components, we evaluate whether the structural similarity formula is appropriate or not. The first inter-connected component is a group of vertices that around vertex 1 and vertex 2 . As mentioned before, vertex 1 is club's instructor, the vertices in the first inter-connected component are members who have good relationship with the instructor. The second inter-connected component is a group of vertices that around vertex33 and vertex34. Vertex34 is the administrator of the club, they are another faction of the club. In Figure 3(b) you may think the second inter-connected component vertexCvertex connections are not dense. But referring to Figure 3(a) the original network, you can find that their vertexCvertex connections are actually dense. The third inter-connected component is an independent faction. They connect to each other closely and they don't have any relationships with other vertices but vertex 1 . It's reasonable that they are distinguished. The fourth inter-connected component, we can find a loop of three vertices. It's a typical local community structure.

But delete edges once may not be enough. For Karate network, if we set the threshold $\alpha=0.48$, after edges deleting, we get networks shown in Figure 4 which has two inter-connected components and seven isolate vertices. The left inter-connected components is obviously a coarse-grained division. It can be divided into two inter-connected components by delete the edge between vertex 3 and vertex 9 . The threshold differs by only 0.02 from threshold mentioned above, but the partition is much worse. Under the circumstances, the LED process can make sure our algorithm obtain good initial communities with wider range of $\alpha$.

\subsection{Overlapping Vertices Detection}

After the Loop Edge Delete Process we get several inter-connected components and many isolated vertices. We treat the connected components as initial communities. Between initial communities, we detect the overlapping vertices. First of all we summarize features of overlapping vertices. The overlapping vertex is the vertex which belongs to several communities at the same time. Overlapping vertices are usually hub vertices as well. They closely connect 


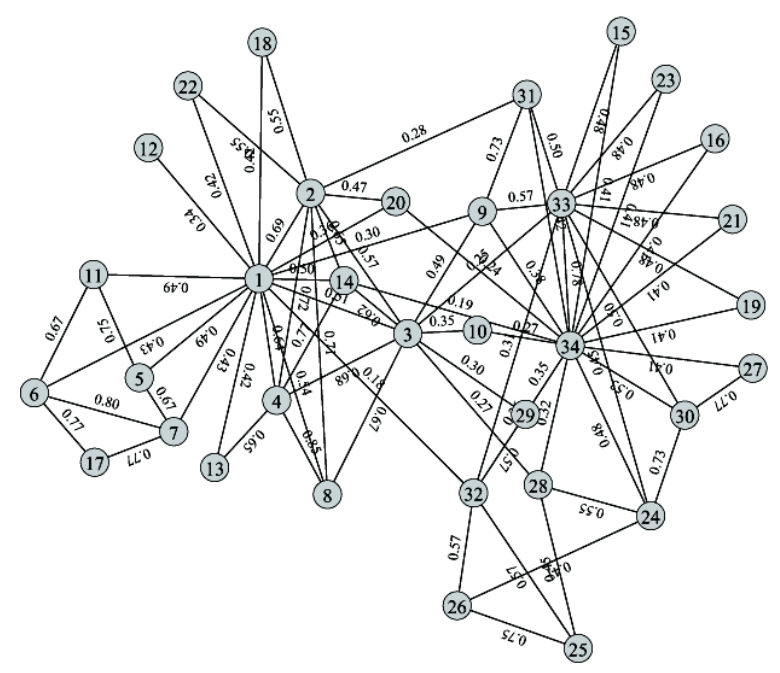

(a)

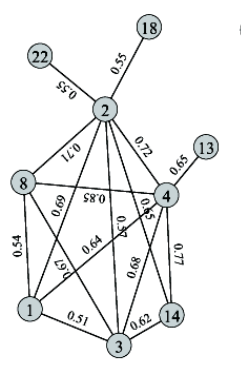

(10)

(23)

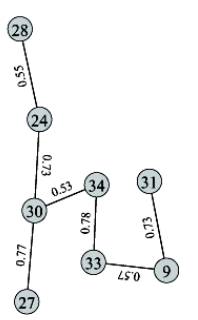

(21)

(12)

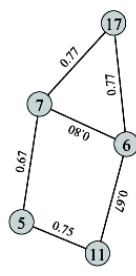

(19)

(15)

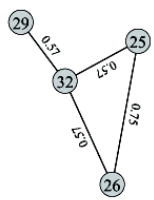

(20)

(16)

(b)

Figure 3: (a)Karate Network: 34vertices, 78edges with structural similarities as weights. (b)Karate Network deleted edges weights below 0.5

to several communities. The overlapping vertex connects to each community with different connection strength while they often have one biased community. In other words, the overlapping vertex connects to each community with different structural similarity. So one community will split into two or more communities around the overlapping vertex and the overlapping vertex will be divided to its most favor community.

The Loop Edge Delete Process has obtained initial communities. We inspect this process in reverse of edges delete order. We find the edge which cause one community splits into two communities when it is deleted. This kind of edges are called bridge edges by us. We already know that the overlapping vertices is around the community split points. We need a method to judge whether a vertex is overlapping vertex or not. We think vertexCvertex connected densely in a community is key feature of community structure. So we adopt average degree of the community[35] which is based on the concept of partition density to evaluate a vertex is a overlapping vertex or not. The definition of average degree is

$$
A D(c)=2 * \frac{|E(c)|}{|c|}
$$

Where $c$ is a community, $E(c)$ is the number of internal edges in the community, the internal edges are the edges that both vertices in the community $c$, and $|c|$ is the number of vertices in $c$. If a vertex adding to the community makes $A D(c)$ increase, we suppose that the vertex contributes to the community. So it should be assigned to this community at the same time which makes it an overlapping vertex.

For examples, in Figure 1, the bridge edges is $e(4,7)$ and $e(5,7)$, for the left community: $A D(c 1)=2 *(10 / 6)=$ 10/3 and the right community: $A D(c 2)=2 *(13 / 7)=26 / 7$. If we add vertex4 to the right community: $A D^{\prime}(c 2)=$ $2 *(14 / 8)=7 / 2<26 / 7$, vertex 4 is not an overlapping vertex. If we add vertex 5 to the right community: $A D^{\prime}(c 2)=$ $2 *(14 / 8)=7 / 2<26 / 7$, vertex 5 is not an overlapping vertex. If we add vertex 7 to the left community: $A D^{\prime}(c 1)=$ $2 *(12 / 7)=24 / 7>10 / 3$, vertex 7 is an overlapping vertex.

\subsection{LED Algorithm}

The process of LED: firstly, transfers the structure relationship between vertices to the their weight; Then, delete the edges in the graph below the threshold $\alpha$ which is specified manually (The problem of parameter specifies will be

discussed in Section 4). LED continuously repeat the above process until no edge is deleted. Secondly, LED gets initial 


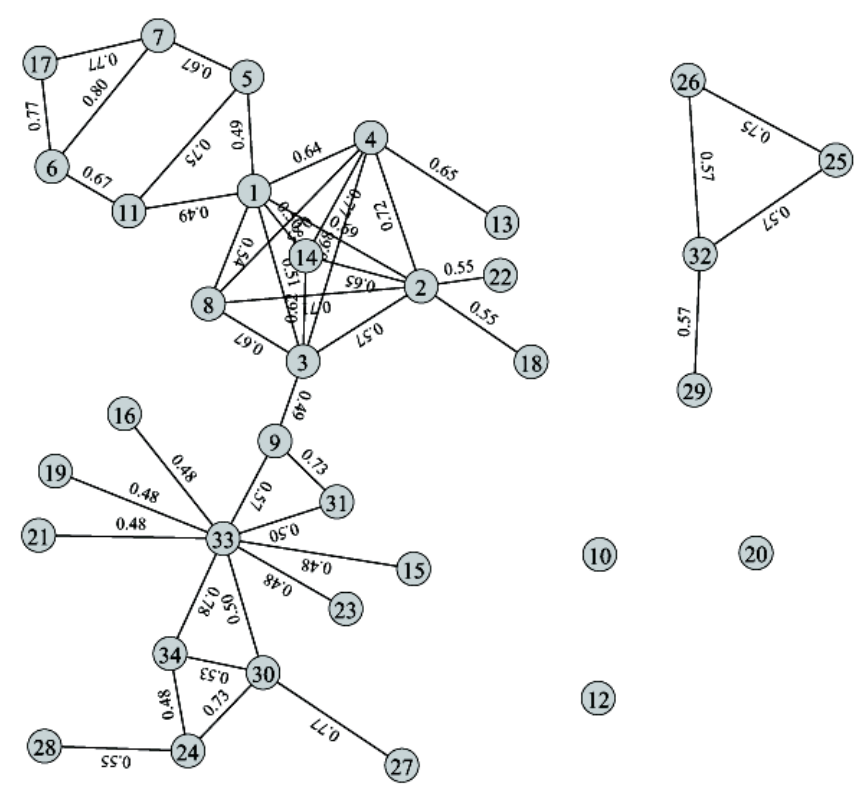

Figure 4: After the edges delete. Threshold $\alpha=0.48$

communities from the graph by identifying the inter-connected components. Thirdly, LED finds overlapping vertices by tracing the deleted edge which cause one community splitting to two communities. Finally, isolated vertices are connected the to the nearest community (with the maximal structural similarity).

The pseudo code of our algorithm is presented in LED Algorithm.

In which, Line 1 defines a current graph pointer and assign it point to G. Line 2 defines a edge list which stores the deleted edges each loop. Line 4 to Line 14 is the Loop Edge Delete Process. Compute structural similarity of the whole graph and remove edges weighted less than $\alpha$ in graph. Push the deleted edges into edge list which will be used in the overlapping vertices detection state and initial communities extension state. This process loop execution until no edge is deleted. Line 15 traverses graph to label all connected components and isolated vertices. Line 16 to Line 20 identifies overlapping vertices by finding the deleted edges which cause one community split to two communities. In Line 21, isolated vertices are connected to the initial community with highest Structural Similarity.

\section{Complex Analysis And Parameter Selection}

\subsection{Complex Analysis}

In this section, we analyze the time complexity of LED algorithm. Let us assume that the given graph $G$ has $m$ edges, $n$ vertices and the average degree is $k$. LED first executes Loop Edges Delete process. For each loop, LED traverses edges of current graph once and for each edge computes the structural similarity of the two vertices. The time complexity is $O(\mathrm{~km})$. Removing edges weighted $<\alpha$ costs $O(\mathrm{~m})$. Thus the Loop Edges Delete process running time is $O(k m+m)$ for each loop. In general, the loop only has 2 or 3 iterations. Its iteration number can be seen as a constant.

Label each inter-connected components as a initial community needs traverse vertices of the graph once which costs $O(n)$. Identifying overlapping vertices need check every deleted edge. The number of deleted edges must be lower than $m$, so the running time is lower than $O(m)$. Attaching the isolated vertices to the initial community with highest Structural Similarity needs check every isolated vertex. The number of isolated vertex may at most be $n$, so the maximum time complexity is $O(k n)$. In summary, the time complexity of our algorithm LED is $O((k c+c+1) m+(k+1) n)$ 


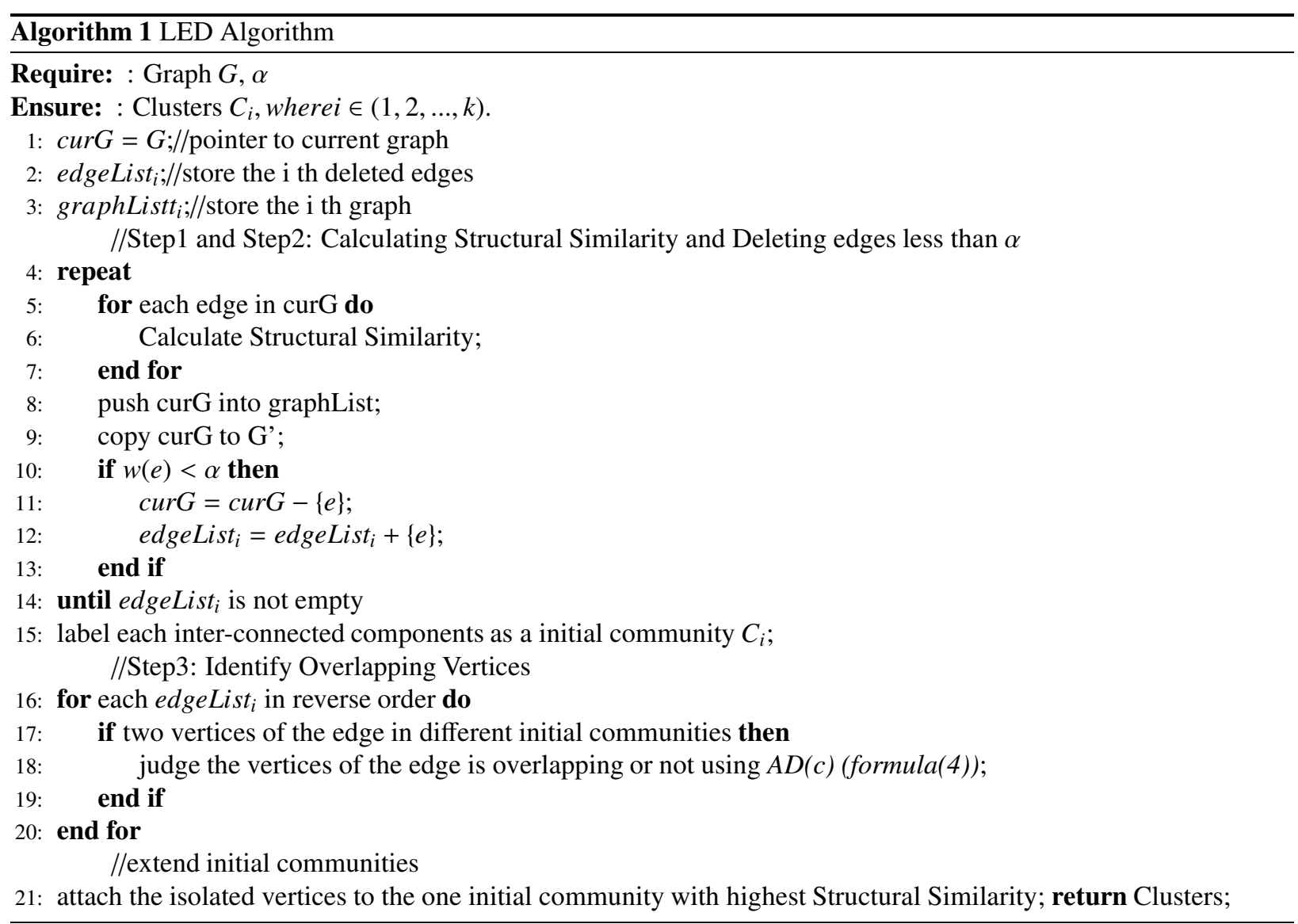

in which $c$ is loop number of Loop Edges Delete process. The time complexity of several overlapping community detection algorithms are shown in Table 1.

\subsection{Parameter Selection}

LED algorithm needs manually setting a parameter $\alpha$ which is the threshold of deciding which edges to delete. The parameter selection concerns the precision of algorithm. The similarity function is sensitive to the threshold $\alpha$. But we adopts The Loop Edges Delete Process, this process can efficiently reduce the similarity function's sensitivity to threshold.

\subsubsection{Measure Quality of Parameter Selection}

How can we say a community detection is good? Modularity is one measure of the structure of networks which measures the quality of community detection. It is first proposed by Girvan and Newman[1]. Networks with high modularity indicate that it is connected strongly within communities and connected sparsely in different communities. The communities which community detection algorithm detects has larger modularity means the detection better. In real networks, modularity usually falls in the range from 0.3 to 0.7 . Modularity is used in many community detection algorithms as objective function. Formula of modularity measure is

$$
M=\sum_{i}\left(e_{i i}-a_{i}^{2}\right)=T r e-\left\|e^{2}\right\|,
$$

where $e_{i i}$ is the fraction of all edges in the network that have both vertices in community $i, a_{i}$ is the row (or column) sums $a_{i}=\sum_{j} e_{i j}$, Tre $=\sum_{i} e_{i i}$. 
Table 1: Time complexity of other overlapping community detection algorithms

\begin{tabular}{ll}
\hline Algorithm & Time Complexity \\
\hline LED & $O((k c+c+1) m+(k+1) n)$ \\
CPM[10] & $O\left(n^{3}\right)$ \\
CONGA[11] & $O\left(m^{3}\right)$ \\
CONGO[13] & $O\left(m \log m+m^{2 h+2} / n^{2 h+1}\right)$ \\
Fuzzy Clustering[17] & $O\left(g n^{2}\right)$ \\
\hline$n$ is the number of nodes, $m$ is the number of edges. \\
$k$ is the average degree. \\
$k<<m, k<<n, m=n * k$. \\
$h$ is a parameter of CONGO to control the scope of \\
local betweenness may be calculated. \\
$g$ is the number of cliques. \\
\hline
\end{tabular}

\subsubsection{Recommended Way to Find Optimal Parameter}

We run our algorithm for every possible threshold $\alpha$ on Newman's Karate network and College Football networks. As shown in Figure5(a)(b), we can figure out that our algorithm is not so sensitive to the threshold $\alpha$. For Karate network with $\alpha$ ranging from 0.32 to 0.78 , we have modularity greater than 0.35 , while the maximum modularity is 0.4155 . For Football network with $\alpha$ ranging from 0.32 to 0.58 , we have modularity greater than 0.5 , while the maximum modularity is 0.601 . We deduce that it is the Loop Edges Delete Process reduce the sensitivity of the threshold $\alpha$. When the threshold $\alpha$ is small, the subsequent edges deleting process makes the network separated enough. When the threshold $\alpha$ is big, the first edges deleting process may delete enough edges and the subsequent edges deleting process doesn't delete any edges. So with a large range of $\alpha$ our algorithm can obtain acceptable community detection results.

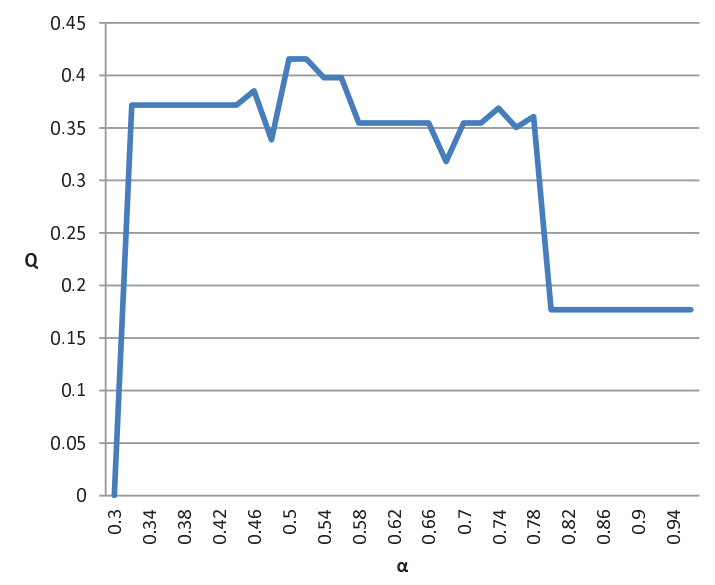

(a)

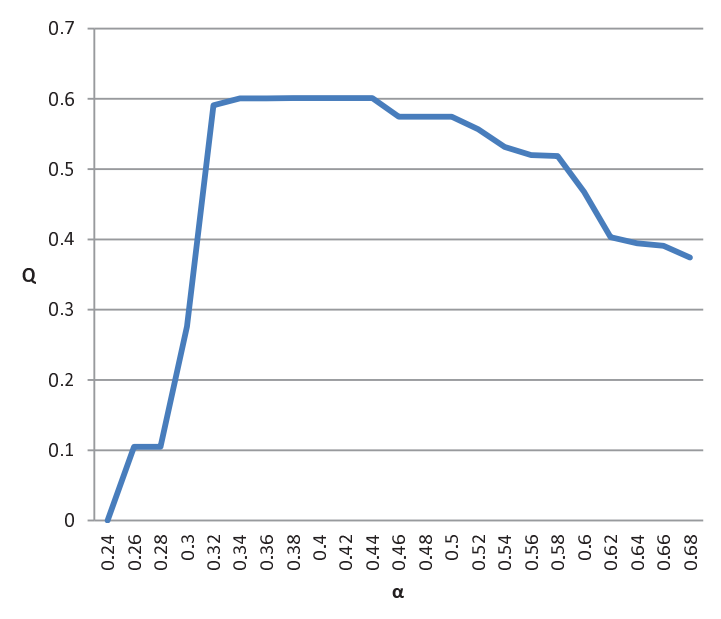

(b)

Figure 5: (a)Karate network's modularity for every possible threshold. (b)Football network's modularity for every possible threshold.

Now we discuss the relationship between the optimal $\alpha$ and the properties of network which can help us narrow 
down the range of $\alpha$. According to our structural similarity formula, the weighs of edges depend on the network is dense or sparse. The average cluster coefficient[39] and the average degree are the metrics that show whether a network is dense or not. The average cluster coefficient of Karate network is 0.588 while the average cluster coefficient of Football network is 0.403 . Karate network is much denser than Football network. Vertex-vertex is likely to have more common neighbors in Karate network. So, the optimal threshold of Karate network is higher than that of College Football network. The average degree of Karate network is 2.294 while the average degree of Football network is 5.33. The average degree of Football network is more than twice as the average degree of Karate network. According to formula(2), networks with larger the average degree are more likely have smaller structural similarity which contributes to the difference of optimal threshold between the two networks.

The properties of network can guide us to find the optimal threshold $\alpha$, but the quantitative relation between them is too complex to determine. An efficient and accurate way to find the optimal $\alpha$ is important for our algorithm, we suggest an empirical and enumerate way to find the optimal $\alpha$. For normal networks $\alpha$ ranging from 0.3 to 0.7 , our algorithm can obtain acceptable community detection results. As discussed in previous paragraph the properties of network can help us narrow down the try range of $\alpha$. There is infinite numbers in [0.3,0.7], but in our experiment the change less than 0.01 is noneffective. For example, in Karate network $\alpha$ in $[0.32,0.44]$ has the same modularity. Observed from the curve in Figure5(a)(b), the optimal $\alpha$ is always corresponds to an $\alpha$ value that is near the middle of its all possible values. To find the $\alpha$ obtaining maximum modularity, we just need to test a portion $\alpha$ of all possible values. Because LED algorithm is efficient, this solution should be appropriate for most networks.

\section{Experiment}

In this section, we evaluate our algorithm using both synthetic and real social networks. We use the synthetic networks to test the efficiency of our algorithm. We use the real social network to test the effectiveness of our algorithm. We implemented LED in C++ based on the SNAP[40](Stanford Network Analysis Platform) which is a general purpose network analysis and graph mining library. All the experiments were conducted on a PC with a $2.9 \mathrm{GHz}$ i7 processor and $4 \mathrm{~GB}$ of RAM.

\subsection{Efficiency}

To evaluate the time complexity of our algorithm LED, we generate 23 graphs with the number of vertices ranging from 1,000 to 1,500,000 and the number of edges ranging from 2,998 to 4,500,000. We generates the small-world graph using the Watts-Strogatz model[22]. SNAP implemented the small-world graph generator. The following parameters need to be seted: $\mathrm{n}$ (number of vertices), $\mathrm{k}$ (each vertex is connected to $k$ nearest neighbors in ring topology), $\mathrm{p}$ (rewiring probability). We set $k=3$ and $p=0.1$ for all 23 graphs.

The performance of our algorithm is compared with FastModularity[41], CPM[19] and CONGO[27]. FastModularity is originally proposed for very large networks. It is an optimization of GN algorithm[1] in time complexity. It is an agglomerative hierarchical algorithm working in an greedy way. FastModularity regards every vertex as a community and then joins two communities at each iteration. The two communities are selected based on the idea of maximizing modularity increment. The result is described by the dendrogram. The time complexity of FastModularity is $O(m d \log n)$, where $m$ is the number of network's edges, $n$ is the number of network's vertices and $d$ is the depth of the result dendrogram. FastModularity can not detects overlapping communities while CPM can. As mentioned above CPM is a famous overlapping community detection algorithm whose time complexity is $O\left(n^{3}\right)$. CONGO is a much more efficient algorithm derived from GN algorithm by calculating local betweenness and it can also detect overlapping communities. All these three baseline algorithms are representative.

Figure 6 shows the running time for LED and FastModularity on the 20 synthetic graphs. As we can see, our algorithm is linear while FastModularity is quadratic. The running time of our algorithm increases smoothly and slowly with the increase number of vertices. The running time of FastModularity increases much more fiercely than our algorithm. It means that our algorithm scales better for very large graphs. For the synthetic graph with 1,000 vertices, the running time of our algorithm and FastModularity are 0.33 second and 0.47 second, respectively. They are basically of the same order of magnitude. For the synthetic graph with 20,000 vertices, our algorithm runs 69 seconds while FastModularity runs 795 seconds. CONGO is the most efficient baseline algorithm. It handles the network with 500,000 vertices within 1010 seconds while LED algorithm completes in 208 seconds. 


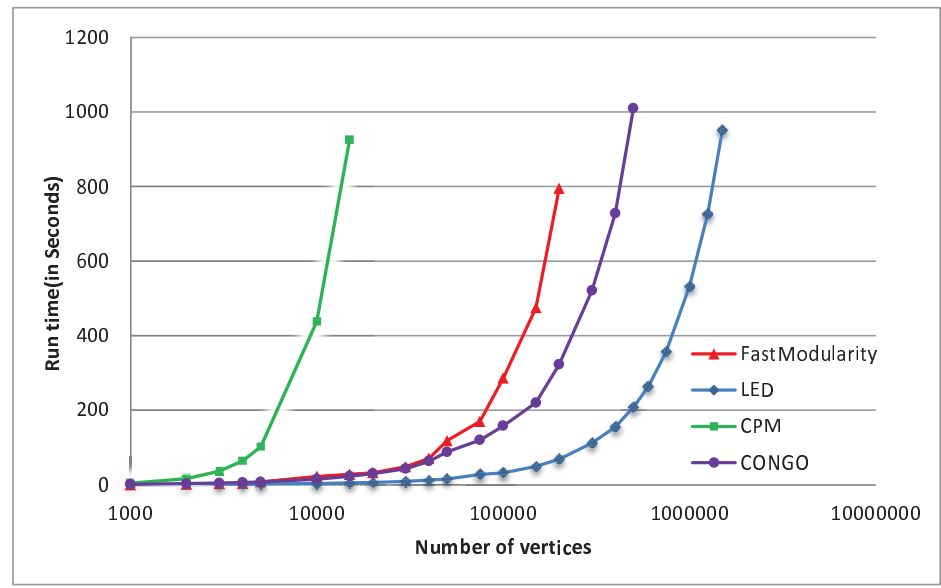

Figure 6: Running time for LED, FastModularity, CPM and CONGO

\subsection{Effectiveness}

Evaluating the effectiveness of community detection is always a hard point. We estimate the the quality of community detection by computing the partition's modularity. Modularity is widely used as a part of community detection algorithms [1, 11, 41] or to evaluate the performance of community detection algorithms[25, 11, 42]. GN algorithm may be the most accurate community detection algorithm, so in this section we choose GN algorithm as our baseline algorithm.

\subsubsection{Karate Club}

We run our algorithm and other competitors on the Karate network. Karate network is a classic dataset which is cited by many algorithms[1, 11, 43]. In section 3.1, we have introduced the Karate network. Newman's work[1] gives the artificial categorization of the Karate network shown in Figure 7(a). The vertices circle around administrator of the club are represented as yellow ellipses while the blue ellipses represent the vertices which are associated with the club's instructor. We run our algorithm on Karate network and get the community detection result as shown in Figure 7(d). Our algorithm divides the network into four communities which are represented as blue, yellow, red and green ellipses and four overlapping vertices which are represented as brown box. First we compare the accuracy of community detection with GN algorithm[1] then we analysis the overlapping vertices our algorithm finds comparing with overlapping vertices found by another algorithm based on community core.

GN algorithm may be the most accurate community detection algorithm, through it is time-consuming. Community detection result of GN algorithm is shown as Figure 7(b). GN divides the network into five communities. The result of GN algorithm is similar to ours. The Modularity of GN algorithm's result is 0.4012 while the Modularity of our result(ignoring the overlapping vertices, take the original divided community as its community) is 0.4155 . Modularity is not the gold standard of community classification quality, but it is a fairly reliable reference standard. Karate network is a quiet small network, so we can artificially analysis. Referring to Newman's artificial categorization in Figure 7(a), our algorithm gives more detailed classification and only vertex 10 is misclassified. Is vertex 10 misclassified? We don't believe so. The vertex 10 connects to vertex 34 and vertex 3 with one edge, respectively. Apparently, vertex 34 and vertex 3 aren't in the same community. We think vertex 10 should be close to the vertex with less degree based on the intuition that a man with many friends his every friendship will be lighter. It just like his friendship is diluted. A small group trends to have more cohesiveness. The degree of vertex 34 is 17 while the degree of vertex 3 is 10 , so we think vertex 3 is a more closer friend to vertex 10 . We just scrutinize the problem from the structure, maybe Newman considered the background that vertex 34 is the administrator of the club, he is obviously more attractive to vertex 10 . GN algorithm can't decide which community the vertex 10 belongs to, so the vertex alone is a community. 


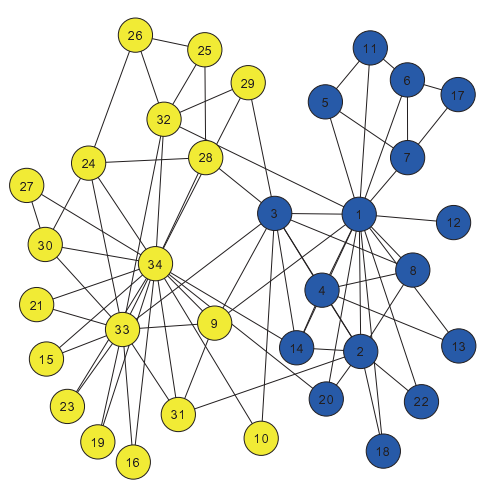

(a)

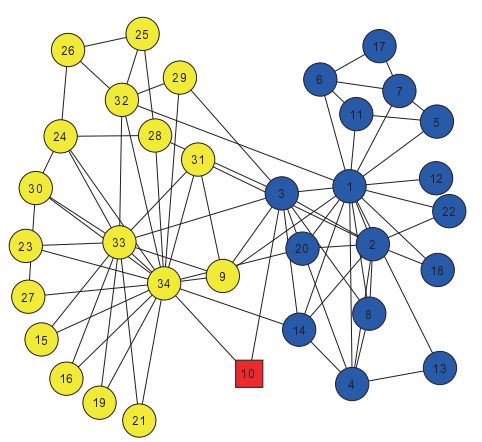

(c)

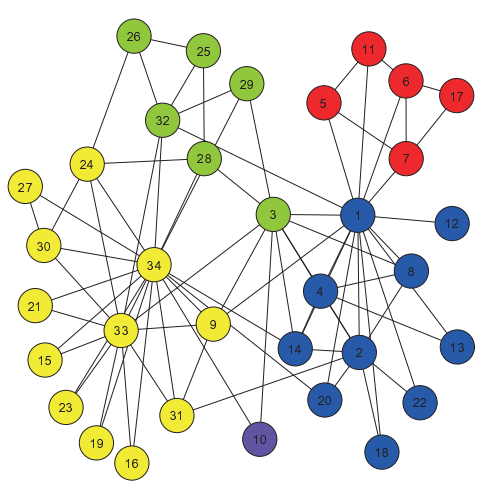

(b)

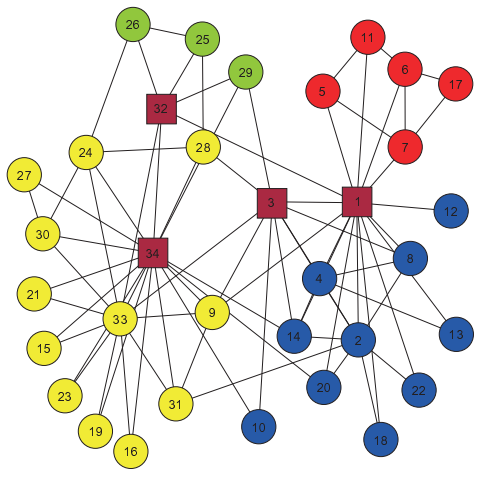

(d)

Figure 7: (a)Community detection result giving by Newman. (b)Community detection result of GN algorithm. (c)Community detection result of Algorithm Based on Community Cores. (d)Community detection result of LED algorithm.

But we think a isolated vertex forming a community is meaningless. The vertex 3 is misclassified by GN algorithm which is mentioned in Newman's paper. The vertex 3 misclassified directly causes vertex 28 misclassified.

Figure 7(c) shows the result of a overlapping community detection algorithm[44] based on community cores. It finds the max-clique as the initial communities and then use an metric named connecting degree to merge communities. At last, it runs a community extension process judging whether a border vertex is an overlapping vertex. Its community detection is the same with the artificial categorization and identifies vertex 10 as the overlapping vertex. Vertex 10 is a normal vertex of the network and its degree is only 2 . Vertex 10 connects to the two communities with same week strength. Identifying it as an overlapping vertex is meaningless, actually, assigning it to either community is fine. Our algorithm identifies four overlapping vertices including the administrator and instructor of the club, each of them is an important vertex to the network. Vertex 1 is a typical overlapping vertex which belongs to red community and blue community at the same time and the red community and blue community have no connections except vertex1. We also run CPM algorithm[19] on the Karate network, but it can't give reasonable community detection result.

\subsubsection{College Football}

The college football network is first proposed by Newman[1]. It is a network with 115 teams(vertices) and 613 edges which is divided into 12 conferences including an independent conference. Matches inside the conference is more than outside the conference, so the network has obvious community structure. It is a labeled network, so we evaluate our algorithm by comparing our result with the labels. Our algorithm divides the network into 12 communities, but there is a little difference with the labeled conferences. The result of our algorithm is shown in Figure 8. It is 


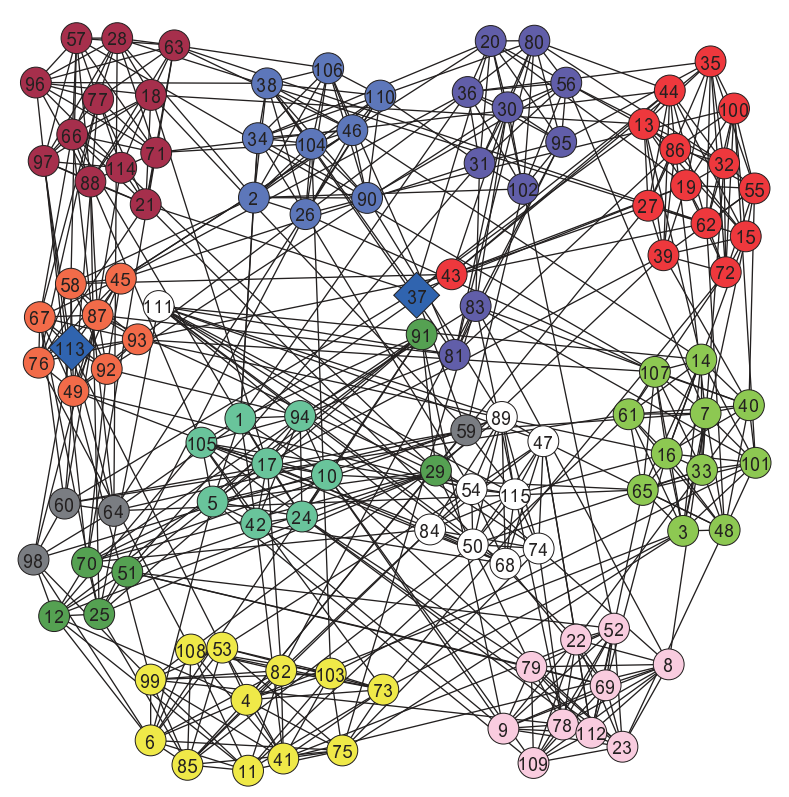

Figure 8: Community detection of LED algorithm on college football network

a special representation that the location gathering is community labels while the colors of the vertices are detection results of our algorithm. Teams of independent conference are spread to other three conferences by our algorithm, the Sun Belt conference is split into two and three other teams are misclassified. The result of GN algorithm is shown in Fig.9 with the same representation. Next paragraph, we will explain why our algorithm makes these "mistakes".

It should be no surprise that the teams of independent conference (vertex 37 , vertex 43 , vertex 81 , vertex 83 , vertex 91 ) are spread to other conferences. The GN algorithm also divides the five independent teams into other communities which are different from ours partly. Our algorithm and GN algorithm both split the Sun Belt conference into two. Vertex 60 , vertex 64 and verte 998 connect to other green vertices with only one edge between vertex 98 and vertex 12 . In terms of the structure, they should be divided as an independent community while GN algorithm merges these three vertices into the Southeastern conference. Our algorithm and GN algorithm both misclassified the three vertices: vertex 29 , vertex 59 and vertex 111 . But in terms of the structure, the misclassified vertices all don't connect to their labeled conferences closely. For an extreme example, vertex 111 has no edges connecting to Conference USA which is the conference vertex 111 labeled. The Modularity of GN algorithm's result is 0.5995 while the Modularity of our algorithm's is 0.601 . We almost have the perfect community detection. The college football network isn't a social network, every vertex have equal status. Overlapping community structure is not obvious in this network. So, our algorithm only finds two vertices which are represented as diamonds. One of them is an independent team. It means the overlapping vertices identified by our algorithm are precise.

\subsection{3. $C-D B L P$}

C-DBLP (http://www.cdblp.cn/) is a system developed by Web and Mobile Data Management Lab (WAMDM), Database Group, Renmin University of China. Its goal is to build a database system of Chinese literature based on the author center. The system provides users with authoritative paper data and convenient query service. It gathers Chinese literature data of Chinese authoritative journals and conferences. WAMDM released four C-DBLP datasets on the web site named Datatang (http://www.datatang.com/data/42404) on July 9, 2012. The four datasets involving fields of computer, economic, law and physics, the specific details are shown in Table 2[45].

We apply LED algorithm to these four datasets using method mentioned in Section 4 to select the threshold. The parameter we select and modularity of the community detection results are shown in Table 3 . We compare our algorithm with GN algorithm which is maybe the most accurate algorithm based on modularity. The modularity of 


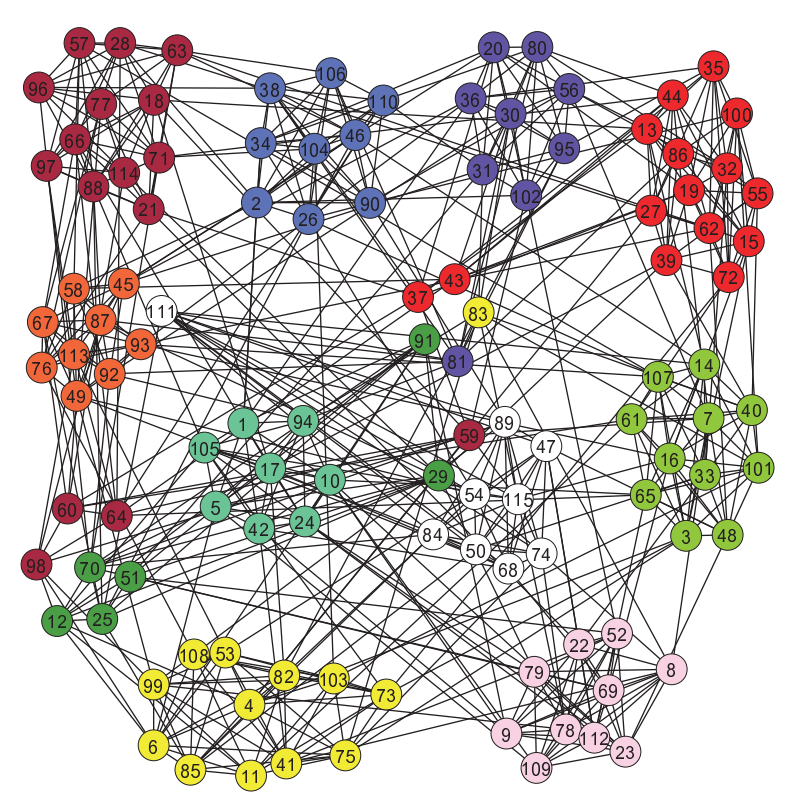

Figure 9: Community detection of GN algorithm on college football network

Table 2: Dataset Statistics of C-DBLP

\begin{tabular}{ccccccc}
\hline Dataset & Vertices & Edges & Average Degree & Diameter & Clustering Coefficient & Average Path Length \\
\hline Computer & 1000 & 2443 & 4.886 & 12 & 0.444 & 5.142 \\
Economic & 1000 & 1435 & 2.87 & 27 & 0.297 & 11.039 \\
Law & 1000 & 2423 & 4.846 & 19 & 0.363 & 6.27 \\
Physics & 1000 & 5051 & 8.102 & 13 & 0.585 & 5.23 \\
\hline
\end{tabular}

LED and GN on the four datasets are shown in Table3 and Table 4, from these two tables we can see that our algorithm have almost same accurate community detection quality with GN algorithm which has the state of art veracity while our algorithm is almost 300 times faster than GN algorithm. We will take computer dataset as an example to discuss the community detection results of our algorithm.

The communities our algorithm finds on computer dataset are shown with different colors in Figure 10. There are 93 communities our algorithm finds, but the Pajek (the graph visualization software) most supports forty colors. Two communities may share one color, but we separate them locally. The overlapping vertices are represented as diamonds. There are two kinds of overlapping vertices, one is obviously biased to one community, the other connects to every community with roughly same strength. The previous kind of overlapping vertices is the majority. For example, in the academic world, a scholar may interests in many fields of research, but he(she) can only be proficient in one or two fields of research.

For example, the grey colored community in the the lower right corner of Figure 10 . The small community has four members and an overlapping vertex, while the four members form one quadrangle and two triangle and the overlapping vertex has an edge to each member of the community. The communications with other communities all pass through the overlapping vertex and it connects to other seven different communities. There is another example. The orange colored community in the the upper left corner of Fig.10. The community consists of seven members and one overlapping vertex. The overlapping vertex is obviously a leader of the research group. There are 15 edges coming from the community while 10 edges coming from the overlapping vertex. This means our algorithm has found 
Table 3: Community Detection Results of LED Algorithm on C-DBLP Datasets

\begin{tabular}{cccccc}
\hline Dataset & Threshold & Number of Communities & Modularity & Number of Overlapping Vertices & Run Time(s) \\
\hline Computer & 0.445 & 93 & 0.65 & 99 & 0.33 \\
Economic & 0.475 & 27 & 0.824 & 64 & 0.30 \\
Law & 0.495 & 183 & 0.653 & 113 & 0.34 \\
Physics & 0.412 & 59 & 0.741 & 82 & 0.38 \\
\hline
\end{tabular}

Table 4: Community Detection Results of GN Algorithm on C-DBLP Datasets

\begin{tabular}{cccc}
\hline Dataset & Number of Communities & Modularity & Run Time(s) \\
\hline Computer & 80 & 0.642 & 92 \\
Economic & 29 & 0.832 & 86 \\
Law & 167 & 0.642 & 94 \\
Physics & 43 & 0.745 & 98 \\
\hline
\end{tabular}

the right overlapping vertex who is in charge of the research group and communicates with other research groups.

There is plenty of other interesting information that we can extract from the community detection results. If we visualize the community detection result of Economic dataset, you can find the vertices forming long lines with some triangles or quadrangles. That may because economists prefer cooperating in pairs. The Law network has a lot small communities which also causes number of overlapping vertices increasing. That may because lawyers work in small groups. The physicists must work in a big group and cooperate with each other often. Because the Physics network has less community number and larger communities among which the largest community has 140 members.

\section{Conclusions}

Community detection is a fundamental task in many fields of science and engineering. Many algorithms have been proposed to solve this problem. Many researchers focus on modularity. The algorithms based on modularity succeed in detecting high quality community in different networks including social and biological networks. There are lots of other successful examples. But they all based on an assumption that one vertex must and only belong to one community. They fail to identify overlapping vertices which play an important role in complex network.

In this paper, we proposed an algorithm named LED(Loop Edges Delete) to detect communities and overlapping vertices. It is a very accurate algorithm, at the same time, it is efficient enough to handle networks with millions of vertices. We represent how two vertices connect by inspecting their common neighbor. In social networks, two people who share more friends in common are more likely to be close friends. There are different kinds of users in the social networks. Specifically in C-DBLP there are authors who are outsider(papers only have one author), and there are also authors who cooperate with other authors of different search fields widely.

We apply LED to real world networks including grouping people on the Karate club personal relationship network, finding conferences using the College Football schedule and discovering research groups from the C-DBLP datasets. In addition, we compared LED with other algorithms including FastModularity, GN algorithm and an Algorithm Based on Community Cores in aspects where they have advantages. LED is a much more faster algorithm than FastModularity, is equal with even better than GN algorithm in the aspect of precision and finds more meaningful and useful overlapping vertices than Algorithm Based on Community Cores.

In future, we plan to extend LED algorithm to dynamic network. In dynamic network, we will study the transformations of structure and functions of networks. 


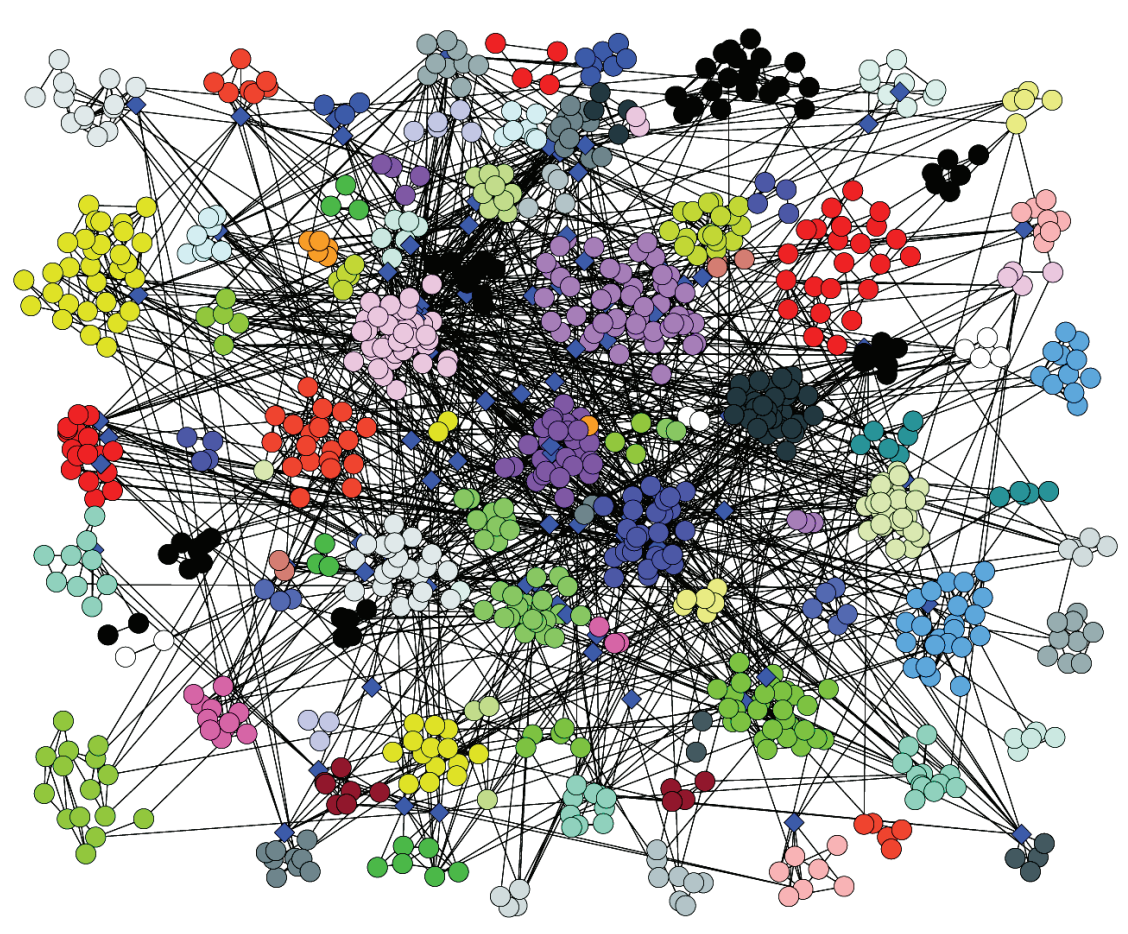

Figure 10: Community detection result of computer dataset by LED algorithm

\section{Acknowledgments}

This work was supported in part by National Science Foundation of China (No.61572259, 61173143), Special Public Sector Research Program of China (No. GYHY201506080), and was also supported by PAPD. The authors extend their appreciation to the Deanship of Scientific Research at King Saud University for funding this work through research group no. RGP-VPP-264.

\section{References}

[1] Girvan M, Newman ME. Community structure in social and biological networks. Proceedings of the National Academy of Sciences;99(12): 7821-6. 2002

[2] Nawaz W, Khan K U, Lee Y K, et al. Intra graph clustering using collaborative similarity measure. Distributed and Parallel Databases, 33 : $1-21,2015$.

[3] A.R. Griffing, B.R. Lynch, E.A. Stone. Structural properties of the minimum cut of partially-supplied graphs. Discrete Applied Mathematics; 177: 152-157, 2014

[4] Yinghua Lv, Tinghuai Ma, Meili Tang et al. An efficient and scalable density-based clustering algorithm for datasets with complex structures, Neurocomputing, 171: 9-22, 2016

[5] Xiang J, Tang Y-N, Gao Y-Y. Multi-resolution community detection based on generalized self-loop rescaling strategy. Physica A: Statistical Mechanics and its Applications;432(0):127-39, 2015.

[6] Kang Y, Yu B, Wang W. Spectral Clustering for Large-Scale Social Networks via a Pre-Coarsening Sampling based Nystrym Method. In: Cao T, Lim E-P, Zhou Z-H editors. Advances in Knowledge Discovery and Data Mining. Lecture Notes in Computer Science. 9078: Springer International Publishing; p. 106-18. 2015.

[7] $\mathrm{Xu} \mathrm{Y,} \mathrm{Xu} \mathrm{H,} \mathrm{Zhang} \mathrm{D.} \mathrm{A} \mathrm{novel} \mathrm{disjoint} \mathrm{community} \mathrm{detection} \mathrm{algorithm} \mathrm{for} \mathrm{social} \mathrm{networks} \mathrm{based} \mathrm{on} \mathrm{backbone} \mathrm{degree} \mathrm{and} \mathrm{expansion.} \mathrm{Expert}$ Systems with Applications, 42(21): 8349-8360, 2015.

[8] Eustace J, Wang X, Cui Y. Overlapping community detection using neighborhood ratio matrix. Physica A: Statistical Mechanics and its Applications;421:510-21, 2015.

[9] Xu X, Yuruk N, Feng Z, et al. Scan: a structural clustering algorithm for networks. Proceedings of the 13th ACM SIGKDD international conference on Knowledge discovery and data mining. ACM, 824-833, 2007.

[10] Sun H, Huang J, Zhang X, et al. IncOrder: Incremental density-based community detection in dynamic networks. Knowledge-Based Systems, 72: $1-12,2014$ 
[11] Newman ME, Girvan M. Finding and evaluating community structure in networks. Physical review E;69(2):026113. 2004.

[12] Rosvall M, Bergstrom CT. Maps of random walks on complex networks reveal community structure. Proceedings of the National Academy of Sciences;105(4): 1118-23, 2008.

[13] Pavani M, Teja G R. Online Clustering Algorithm for Restructuring User Web Search Results. Proceedings of the 3rd International Conference on Frontiers of Intelligent Computing: Theory and Applications (FICTA) 2014. Springer International Publishing, 27-36, 2015.

[14] Sun X, Lin H, Xu K. A social network model driven by events and interests. Expert Systems with Applications;42(9): 4229-38, 2015.

[15] Chen J, Zhang H, Guan Z-H. Epidemic spreading on networks with overlapping community structure. Physica A: Statistical Mechanics and its Applications;391(4): 1848-54, 2012

[16] Bin Gu, Victor S. Sheng, Keng Yeow Tay et al. Incremental Support Vector Learning for Ordinal Regression, IEEE Transactions on Neural Networks and Learning Systems, 26 (7): 1403-16, 2015.

[17] Tinghuai MA, Jinjuan ZHOU, Meili TANG et al., Social network and tag sources based augmenting collaborative recommender system, IEICE transaction on Information and Systems, 98D(4): 902-910, 2015

[18] Ma T, Zhang Y, Cao J, et al. KDVEM: a k-degree anonymity with vertex and edge modification algorithm. Computing 97(12): 1165-1184, 2015.

[19] Palla G, Dernyi I, Farkas I, Vicsek T. Uncovering the overlapping community structure of complex networks in nature and society. Nature;435(7043): 814-8. 2005

[20] Liu X K, Gao N, Wang L H. The MPPT Method and Simulation Study Based on Graph Theory. Mathematics in Practice and Theory, 6: 197-204, 2015

[21] Yong-Yeol A, Bagrow J P, Sune L. Link communities reveal multiscale complexity in networks. Nature, 466(7307):761-764, 2010.

[22] Brian B, Brian K, Newman M E J. Efficient and principled method for detecting communities in networks. Physical Review E Statistical Nonlinear and Soft Matter Physics, 84(3Pt2): 109-134, 2011.

[23] Gregory S. An algorithm to find overlapping community structure in networks. Knowledge discovery in databases: PKDD 2007: Springer; p. 91-102, 2007.

[24] Li A, Li J, Pan Y. Discovering natural communities in networks. Physica A Statistical Mechanics And Its Applications, 436: 878-896, 2015.

[25] Eustace J, Wang X, Cui Y. Community detection using local neighborhood in complex networks. Physica A Statistical Mechanics And Its Applications,436: 665-677, 2015.

[26] Magaia N, Francisco AP, Pereira P. Betweenness centrality in Delay Tolerant Networks: A survey. Ad Hoc Networks;(0). 2015.

[27] Gregory S. A fast algorithm to find overlapping communities in networks. Machine Learning and Knowledge Discovery in Databases: Springer; p. 408-23, 2008.

[28] Du N, Wu B, Pei X, Wang B, Xu L, editors. Community detection in large-scale social networks. Proceedings of the 9th WebKDD and 1st SNA-KDD 2007 workshop on Web mining and social network analysis; 2007.

[29] Shen H, Cheng X, Cai K, Hu M-B. Detect overlapping and hierarchical community structure in networks. Physica A: Statistical Mechanics and its Applications;388(8): 1706-12. 2009.

[30] Lee C, Reid F, McDaid A, Hurley N. Detecting highly overlapping community structure by greedy clique expansion. arXiv preprint arXiv:10021827. 2010.

[31] Magdon-Ismail M, Purnell J. SSDE-Cluster: Fast Overlapping Clustering of Networks Using Sampled Spectral Distance Embedding and GMMs[C], Privacy, Security, Risk and Trust (PASSAT) and 2011 IEEE Third Inernational Conference on Social Computing (SocialCom), 2011 IEEE Third International Conference on. IEEE,756 - 759, 2011.

[32] Zhang S, Wang R-S, Zhang X-S. Identification of overlapping community structure in complex networks using fuzzy c-means clustering. Physica A: Statistical Mechanics and its Applications;374(1):483-90. 2007.

[33] Chen J-L, Hu J-W, Yang C-S. The Bridge Edge Label Propagation for Overlapping Community Detection in Social Networks. Intelligent Data analysis and its Applications, Volume I: Springer; p. 97-102, 2014.

[34] Xie J, Szymanski BK. Towards linear time overlapping community detection in social networks. Advances in Knowledge Discovery and Data Mining: Springer; p. 25-36, 2012.

[35] Cai Y, Shi C, Dong Y, Ke Q, Wu B. A novel genetic algorithm for overlapping community detection. Advanced Data Mining and Applications: Springer; p. 97-108, 2011.

[36] Dice L R. Measures of the amount of ecologic association between species. Ecology, 1945, 26(3): 297-302.

[37] Vorontsov I E, Kulakovskiy I V, Makeev V. Jaccard index based similarity measure to compare transcription factor binding site models. Algorithms for Molecular Biology, 8(1): 23, 2013.

[38] Zhang Z, Wang Z. Mining overlapping and hierarchical communities in complex networks. Physica A: Statistical Mechanics and its Applications;421(0): 25-33, 2015.

[39] Xia Y, Fan J, Hill D. Cascading failure in WattsCStrogatz small-world networks. Physica A: Statistical Mechanics and its Applications;389(6): $1281-5,2010$

[40] http://snap.stanford.edu/.

[41] Clauset A, Newman ME, Moore C. Finding community structure in very large networks. Physical review E;70(6):066111, 2004.

[42] Costa A. MILP formulations for the modularity density maximization problem. European Journal of Operational Research;245(1): 14-21, 2015.

[43] Song Y, Bressan S, Dobbie G. Fast Disjoint and Overlapping Community Detection. Transactions on Large-Scale Data-and KnowledgeCentered Systems XVIII: Springer; p. 153-79, 2015.

[44] Ming-Sheng S, Duan-Bing C, Tao Z. Detecting overlapping communities based on community cores in complex networks. Chinese Physics Letters;27(5): 058901. 2010.

[45] Tinghuai MA, Huan Rong, Changhong Ying et al. Detect structural-connected communities based on BSCHEF in C-DBLP. Concurrency and Computation: Practice and Experience, 28: 311-330, 2016. 